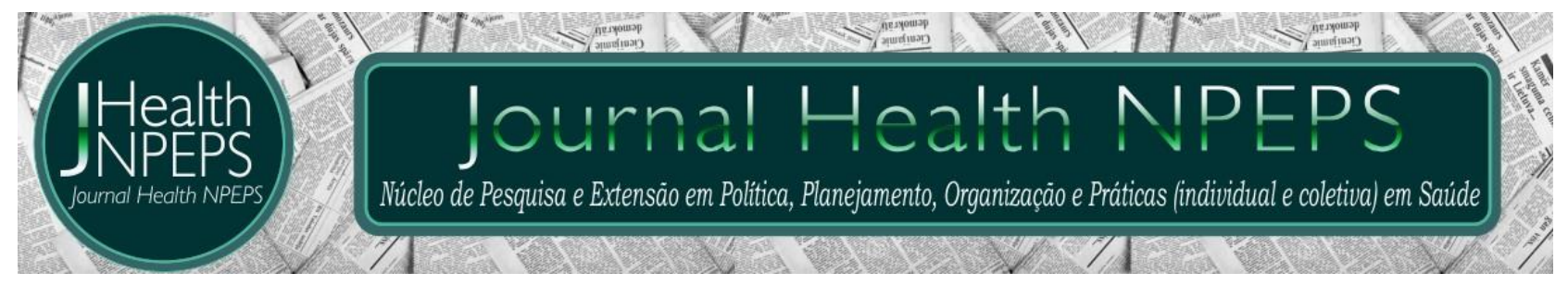

http://dx.doi.org/10.30681/252610102993

ARTIGO ORIGINAL

\title{
Valores terminales, instrumentales y consumo de tabaco en el personal de salud
}

Terminal, instrumental values and tobacco consumption in health personnel

Valores terminais, instrumentais e consumo de tabaco no pessoal de saúde

\section{Carla Sánchez-Serrano ${ }^{1}$, Inés Tenahua-Quitl ${ }^{2}$, Noé Xicali-Morales ${ }^{3}$, Francisco Adrián Morales-Castillo ${ }^{4}$, Maria Luz de Avila-Arroyo ${ }^{5}$, Alejandro Torres-Reyes ${ }^{6}$}

\section{RESUMEN}

Objetivo: identificar la relación entre los valores terminales $e$ instrumentales y el consumo de tabaco en el personal de salud de una institución pública de primer nivel de atención. Método: diseño descriptivo correlacional. La población fue de 165 participantes, los instrumentos fueron una cédula de datos personales y prevalencia de consumo de drogas lícitas y cuestionario de valores de Schwartz y Bilksy. Resultados: el 22\% del personal de salud consume tabaco, edad de inicio 13 años, consumen 1 cigarrillo en un día típico, ingesta mayor del sexo masculino. Se reportan las siguientes prevalencias:global 73.3\%, lápsica 3\%, actual $4.7 \%$ e instantánea $2.4 \%$. El valor instrumental del personal de salud que destacan fue ser capaz, el valor terminal fue una vida excitante. No se encontró relación entre los valores y el consumo de tabaco del personal de salud $(r=-.157, p=0.44)$, tampoco con los valores instrumentales $(r=-$ $.050, p=.591)$. Conclusión: el consumo de tabaco del personal de salud es mínimo y los valores que obtuvieron se encuentran en condiciones para poder brindar la orientación para disminuir el consumo.

Descriptores: Salud Pública; Consumo de Tabaco; Personal de Salud.

\section{ABSTRACT}

\footnotetext{
${ }^{1}$ Enfermera. Maestra. Centro de Salud de San Miguel Tenextatiloyan Zautla, Secretaría de Salud. E-mail: karenaguilar20@outlook.com ORCID ID: https://orcid.org/0000-0003-2172-4967 Autor principal - Dirección de envío: 6 norte número 2204 Barrio de Jesús, San Pedro Cholula, Puebla, México.

${ }^{2}$ Enfermera. Maestra. Profesor Investigador. Benemérita Universidad Autónoma de Puebla, Facultad de Enfermería. Puebla, Puebla, México. E-mail: inesteq@gmail.com ORCID ID: https://orcid.org/0000-0002-3987-8254

${ }^{3}$ Enfermera. Maestro. Profesor Investigador. Benemérita Universidad Autónoma de Puebla, Facultad de Enfermería. Puebla, Puebla, México. E-mail: noexicalimorales@hotmail.com ORCID ID: https://orcid.org/0000-0002-5622-4906 ${ }^{4}$ Enfermero. Maestro. Profesor Investigador. Benemérita Universidad Autónoma de Puebla, Facultad de Enfermería. Puebla, Puebla, México. E-mail: holistic-care21@hotmail.com ORCID ID: https://orcid.org/0000-0002-6772-4690 ${ }^{5}$ Enfermera. Maestra. Profesor Investigador. Benemérita Universidad Autónoma de Puebla, Facultad de Enfermería. Puebla, Puebla, México. E-mail: maluz deavila@hotmail.com ORCID ID: https://orcid.org/0000-0003-4924-1959

${ }^{6}$ Enfermero. Maestro. Hospital General Zona Norte. Secretaría de Salud del Estado de Puebla. Puebla, Puebla, México. E-mail: alexanderreyes20102029@gmail.com ORCID ID: https://orcid.org/0000-0002-4047-4329
}

Este artigo está licenciado sob forma de uma licença Creative Commons Atribuição 4.0 Internacional, que permite uso irrestrito, distribuição e reprodução em qualquer meio, desde que a publicação original seja corretamente citada. 
Objective: identify the relationship between the terminal and instrumental values and the consumption of tobacco in the health professionals of a primary care level of a public institution. Method: correlational descriptive design. The population was 165 participants. The instruments were a personal data and a prevalence of licit drug consumption survey and Schwartz and Bilsky value questionnaire. Results: $22 \%$ of the health professionals consume tobacco, starting at 13 years old, consuming one cigarette in a typical day with higher intake of male. The prevalence reports are: global $73.3 \%$, lapsic $3 \%$, current $4.7 \%$ and instant $2.4 \%$. The most important instrumental value of the health professionals was to be able and the in terminal values was an exciting life. No relationship was found between the values and the tobacco consumption of the health professionals $(r=-.157, p=0.44)$, for the instrumental values there is no relation $(r=-$ $.050, p=.591)$. Conclusion: the tobacco consumption of health professionals is minimal and the values obtained are in conditions to provide guidance to reduce consumption.

Descriptors: Public Health; Tobacco use; Health Personnel.

\section{RESUMO}

Objetivo: identificar a relação entre os valores terminais, instrumentais e o consumo de tabaco na equipe de saúde de uma instituição pública de primeiro nível de atenção. Método: estudo descritivo correlacional. A população foi de 165 participantes e os instrumentos utilizados foram um questionário de dados pessoais e prevalência de consumo de drogas lícitas e questionário de valores de Schwartz e Bilksy. Resultados: 22\% dos profissionais de saúde consomem tabaco, idade de início 13 anos, consomem 1 cigarro em um dia típico, a maior ingesta é mais no sexo masculino. As seguintes prevalências são relatadas: global $73,3 \%$, lapsica $3 \%$, atual $4,7 \%$ e instantâneo $2,4 \%$. 0 valore instrumental do pessoal de saúde que se destacaram foi poder, o valore terminal foram uma vida excitante. Não foi encontrada relação entre os valores e o consumo de tabaco do pessoal de saúde $(r=-.157, p=0,44)$, quanto aos valores instrumentais não há relação $(r=-050, p=0,591)$. Conclusão: 0 consumo de tabaco do pessoal de saúde é mínimo e os valores obtidos estão em condições de orientar a redução do consumo.

Descritores: Saúde Pública; Consumo de Tabaco; Pessoal de Saúde.

\section{INTRODUCCIÓN}

El consumo de tabaco es una de las mayores amenazas para la salud pública, más de 7 millones de personas al año han muerto por esta causa; y de éstas más de 6 millones son consumidores directos y alrededor de 890, 000 son no fumadores expuestos al humo ajeno y $80 \%$ de fumadores en el mundo viven en países de ingreso bajo y medio ${ }^{1}$. Asimismo el consumo de tabaco, es un factor de riesgo común para las cuatro principales enfermedades no transmisibles: cardiovasculares, respiratorias, cáncer y diabetes ${ }^{2}$. Además el tabaco no es un producto más de consumo, si no también el único producto legal que, consumido como lo indican sus fabricantes, mata entre un tercio y la mitad de sus consumidores ${ }^{3}$.

En México se reportan 15.6 millones de fumadores activos de 18 a 65 años que corresponde al $23.6 \%$ de adultos que consumen tabaco, de los cuales 11 millones son 
hombres, 4.6 mujeres y 6.8 fumadores diarios. También la edad promedio de inicio de consumo de tabaco en un día típico es de 20.6 años y fuman hasta 6.6 cigarrillos diarios. La prevalencia global reportada de este consumo es de 40.2\%, lápsica 12.8\%; actual 25.9\% e instantánea 18\%. Por lo tanto el consumo de tabaco contribuye a desarrollar más de cincuenta enfermedades, de las cuales la que presenta la mayor morbilidad es la enfermedad pulmonar obstructiva crónica, que alcanza una tasa de 218 hospitalizaciones por millón de habitantes 4 .

En Puebla se estimó que 31.7\% de la población de 20 años de edad ha consumido más de 100 cigarrillos en toda su vida, con un porcentaje mayor en hombres (53.3\%) que en mujeres (13.9\%), y una razón hombre mujer de 4:1. Por lo tanto, Puebla se ubicó en la posición número 11 (23.2\%) entre todos los estados y estuvo ligeramente arriba de la estimación nacional $23.0 \% 5$.

En otras investigaciones realizadas respecto al consumo de tabaco en profesionales de salud en distintos países entre ellos España, Perú y Bolivia la mayor parte de la población estudiada era del género femenino ${ }^{6-8}$. De acuerdo con los autores ${ }^{6}$ se evidenció que en Lima, Perú 67,2\% del personal de enfermería había fumado una vez en la vida y un 3\% en el último mes. De 132 (64.7\%) entrevistados, 107 (81\%) afirmaron que la edad en que probaron por primera vez el tabaco fue cuando tenían más de 18 años. En otra investigación en profesionales de salud de Cataluña ${ }^{7}$ se obtuvo que $92.8 \%$ de los fumadores inició el consumo antes de los 35 años, además $45.13 \%$ y $26.55 \%$ en el 2001 y 2011 respectivamente refirió fumar entre 11 y 20 cigarrillos al día, diferencia estadísticamente significativa $(p<0,001)$. Otro estudio en el personal de salud La Paz Bolivia ${ }^{8}$, muestra que la prevalencia de hábito de fumar en dicho personal es de $26.9 \%$ y es mayor en el sexo masculino $(30,87)$, sin embargo a exposición más alta fue para el sexo femenino con $57.99 \%$. Otros autores señalan que la prevalencia del consumo de tabaco fue $33 \%$ que los fumadores activos consumen 17,5 cigarrillos al día; $12 \%$ fuman su primer cigarrillo antes de transcurridos 5 minutos desde el momento en el que se levantan de la cama y $26 \%$ entre 6 y 30 minutos después y el comienzo de la ingesta de tabaco fue a los 18 años 9.

Por su parte Maya ${ }^{10}$ menciona que el consumo de tabaco en el personal de salud va en contra del profesionalismo y la ética, ya que estos son responsables de la promoción de la salud y la prevención de enfermedades sin embargo la literatura señala que hay factores de riesgo que contribuyen al consumo de tabaco y destaca el ambiente 
laboral ya que en él se viven situaciones con desgaste psicofísico, con responsabilidad laboral y condiciones de trabajo de riesgo ${ }^{11}$.

Es así que la calidad profesional de salud no sólo depende de los conocimientos y habilidades que desarrolle en el currículo universitario, sino también de los intereses y valores que regulen la actuación del profesional. Consecuentemente, los valores forman parte de los contenidos de enseñanza-aprendizaje que no son competencia exclusiva de los docentes, si no el medio socio familiar y laboral ${ }^{12}$.

Por lo que cobra importancia el concepto de valores ya que estos indican la forma como el ser humano debe comportarse y sirve como punto de referencia para regular si la conducta está a niveles adecuados con respecto a lo establecido, es decir como base para racionalizar la conducta, esto a su vez guía al ser humano a seleccionar conductas frente al consumo de tabaco. Rokeach ${ }^{13}$ clasifica a los valores en terminales $e$ instrumentales los primeros son las metas alcanzadas, los segundos se refieren al comportamiento del personal de salud. En investigaciones se ha determinado que los valores (terminales) placer y vida excitante, así como los valores (instrumentales) el ser atrevido, gozar de la vida curiosa y mente abierta se relacionan con el consumo de tabaco ${ }^{14-17}$. Igualmente se han abordado los valores y se encontró mayor presencia de valores instrumentales que terminales ${ }^{15,16}$.

Teniendo en cuenta que el personal de salud constituye una fuente importante de información e influencia, para lograr cambios favorables en la disminución de los niveles de consumo de tabaco en la población, es importante su participación para transformar el comportamiento negativo en la comunidad ${ }^{15}$. Es así que el objetivo de la investigación fue identificar relación existe entre los valores terminales $e$ instrumentales y el consumo de tabaco en el personal de salud en una institución pública de primer nivel de atención del Estado de Puebla, México.

\section{MÉTODO}

Este estudio fue de tipo descriptivo correlacional ${ }^{18}$. La población de estudio fue de 165 participantes de diferentes categorías laborales, de ambos sexos que laboran en una institución pública de primer nivel de atención del estado de Puebla., de los cuales 39 pertenecieron al personal médico, 80 de enfermería, 17 estomatología y 29 promotores de salud. Para la recolección de los datos se utilizó la cédula de datos 
personales y prevalencia de consumo de drogas lícitas (CDPPCDL) y el Cuestionario de Valores de Schwartz (VAL). La CDPPCDL está constituida por diez ítems y se encuentra conformadas por dos apartados, el primero consta de cinco preguntas las cuales se refieren a los datos personales como edad, sexo, estado civil, escolaridad, categoría laboral, el segundo la conforman cinco preguntas referentes a la prevalencia del consumo de tabaco. En lo que respecta al cuestionario de valores (VAL), elaborado por Schwartz y Bilsky a partir del Rokeach Value Survey, este cuenta con 56 ítems, partiendo de diez dimensiones: hedonismo, logro, poder, seguridad, conformidad, tradición, benevolencia, universalismo, autodirección y estimulación.

Consta de dos subescalas, el primer listado son los valores terminales del 1 al 30 y el segundo listado los valores instrumentales del 31 al 56. Cada ítem es valorado como 1) nada importante, 2) importante y 3) muy importante. El Cuestionario de Valores de Schwartz, ha sido utilizado en varios estudios reportando una confiabilidad de alfa Cronbach .90 y .92 respectivamente ${ }^{14-16}$.

Esta investigación tuvo la aprobación de la Secretaría de Investigación y Estudios de posgrado con el registro SIEP/ME/011/2016. Para la recolección se acudió con los directivos de la Jurisdicción Sanitaria Número 4, para explicarles el objetivo de la investigación y se obtuvo la autorización de la coordinación del departamento de enseñanza quien proporcionó día y hora donde el personal de salud se reunió en la aula de usos múltiples, para invitarlos a participar, explicar los objetivos del estudio y hacer énfasis en el anonimato y confidencialidad de la información, una vez que aceptaron participar se les dio el consentimiento informado, seguido de eso se entregó los instrumentos, y explicó a los participantes que deberán contestar los instrumentos lo más apegado a su realidad, se solicitó de forma amable no dejar alguna pregunta sin contestar, el investigador se mantuvo a una distancia considerable de los participantes por si hubiera preguntas o dudas, al término del llenado de los cuestionarios, se revisaron los instrumentos para evitar dejar preguntas sin contestar, para finalizar se les agradeció su participación y colaboración.

El presente estudio se apegó a lo dispuesto en el Reglamento de la Ley General de Salud en Materia de Investigación para la salud, $2014^{19}$. Se fundamentó en el capítulo Il de acuerdo con el artículo 13 se respetó la dignidad de los participantes con un trato respetuoso, profesional protegiendo así sus derechos, además de hacer entrega del consentimiento informado para autorizar la participación en la investigación. 
Los datos obtenidos se procesaron de manera electrónica mediante el programa estadístico Statistical Package for the Social Sciences (SPSS) versión 21.0 para Windows. La consistencia interna de los instrumentos se determinó a través del Coeficiente de confiabilidad alfa de Cronbach, donde los valores reportó un alfa de Cronbach de .90, en la subescala de valores terminales .81 y en los valores instrumentales .86. Se utilizó estadística descriptiva para obtener frecuencias, proporciones, medidas de tendencia central y de variabilidad. Para determinar la relación del perfil de valores con el consumo de tabaco se utilizó el coeficiente de correlación de Pearson.

\section{RESULTADOS}

De acuerdo con los datos obtenidos $62 \%$ de los participantes correspondió al sexo femenino, la edad promedio fue de 36 años, $D E=8.3$ valor máximo 55 y mínimo 21. El estado civil del personal de salud reportó que 50\% son casados, seguido de solteros con $34 \%$, unión libre $14 \%$ y $2 \%$ son divorciados. Su promedio de años de estudio es de 17.1 años, $D E=2$ con un máximo de 23 y mínimo 12. En lo que se refiere a la categoría del personal de salud $24 \%$ eran personal médico, $49 \%$ de enfermería, $10 \%$ correspondía a estomatología y $17 \%$ promotores de salud.

En lo que respecta al consumo de tabaco, 22\% de los participantes consume tabaco, con un promedio de 1 cigarrillo en un día típico y el inicio fue a los 13 años de edad. En lo que concierne a la descripción de los valores terminales se obtuvo una $\bar{X}=68$ $(D E=12)$, mientras que para los valores instrumentales se halló una $\bar{X}=73(D E=13)$.

En lo que corresponde a la prevalencia del consumo de tabaco en el personal de salud se reporta en la siguiente tabla.

Tabla 1 - Prevalencia del consumo de tabaco. $(n=165)$

\begin{tabular}{lrrrr} 
Prevalencias & Sí & \multicolumn{3}{c}{ No } \\
Global & \multicolumn{1}{c}{$\%$} & $\%$ & $F$ & $\%$ \\
\cline { 2 - 5 } Lápsica & 121 & 73.3 & 44 & 26.7 \\
Actual & 5 & 3.0 & 160 & 97.0 \\
Instantánea & 16 & 9.7 & 149 & 90.3 \\
\hline & 4 & 2.4 & 161 & 97.6 \\
\hline
\end{tabular}

Nota: $f=$ frecuencia,$\%$ = porcentaje.

En lo que se refiere a los valores terminales e instrumentales más frecuentes en el personal de salud se presentan las siguientes tablas 2 y 3. 
Tabla 2 - Frecuencia de los valores terminales del personal de salud que consume tabaco. $(n=165)$

\begin{tabular}{|c|c|c|c|c|c|}
\hline \multicolumn{2}{|l|}{ Nada importante } & \multicolumn{2}{|l|}{ Importante } & \multicolumn{2}{|l|}{ Muy Importante } \\
\hline Valor & $\%$ & Valor & $\%$ & Valor & $\%$ \\
\hline $\begin{array}{l}\text { Tener control en los } \\
\text { demás }\end{array}$ & 44 & Proteger a la nación & 66 & $\begin{array}{l}\text { Una vida excitante } \\
\text { (Experiencias } \\
\text { estimulantes) }\end{array}$ & 78 \\
\hline Pertenecer a alguien & 33 & Derecho de mandar & 64 & $\begin{array}{l}\text { Creer en propio valor } \\
\text { personal }\end{array}$ & 75 \\
\hline Tener cosas materiales & 32 & $\begin{array}{l}\text { Tener el respeto de los } \\
\text { demás }\end{array}$ & 61 & Despreocupación & 59 \\
\hline
\end{tabular}

Nota: $\%$ = porcentaje.

En la tabla se presentan los valores terminales muy importantes para el personal de salud que pertenecen a los valores de estimulación, autodeterminación y tradición.

Tabla 3 - Frecuencia de los valores instrumentales del personal de salud que consume tabaco. $(n=165)$

\begin{tabular}{cccccc}
\hline Nada importante & $\%$ & Importante & $\%$ & Muy Importante & $\%$ \\
$\begin{array}{c}\text { Tener influencia en las } \\
\text { personas }\end{array}$ & 30 & $\begin{array}{c}\text { Evitar acciones } \\
\text { extremas }\end{array}$ & 72 & $\begin{array}{c}\text { Ser capaz } \\
\text { competente }\end{array}$ & 78 \\
$\begin{array}{c}\text { Buscar aventuras } \\
\begin{array}{c}\text { Influir en las } \\
\text { personas }\end{array}\end{array}$ & 64 & Confiable & 78 \\
$\begin{array}{c}\text { Aceptar la parte } \\
\text { de la vida }\end{array}$ & 58 & $\begin{array}{c}\text { Cumplir con los } \\
\text { deberes }\end{array}$ & 77 \\
\hline
\end{tabular}

Nota: $\%$ = porcentaje.

La tabla 3 muestra los valores instrumentales del personal de salud que pertenecen al valor logro, conformidad, benevolencia. También se evidencio que no existe relación entre los valores terminales y la cantidad de cigarrillos consumidos en un día típico $(r=-.157, p=0.44)$.

\section{DISCUSIÓN}

Con respecto a las características sociodemográficas la situación de género de los participantes muestran diferencias porcentuales entre hombres y mujeres, donde las mujeres ocuparon un $62.4 \%$ datos que coinciden con otros autores ${ }^{7-9,15}$, donde presentó un alto porcentaje el género femenino, esto podría ser porque en la mayoría de los países es más alta la población de mujeres que hombres.

En relación al consumo de tabaco del personal de salud, se evidenció que sólo $22 \%$ consume tabaco, estos datos son afines con otros estudios ${ }^{8-10}$, donde el consumo de tabaco presenta porcentajes mínimos, esto es el reflejo de las estrategias contra las adicciones emitidas por los programas conformados por los Consejos Estatales contra las 
adicciones, en estricto apego al pacto federal asentado en la Constitución Política de los Estados Unidos.

El promedio de consumo de cigarrillos en un día típico es de 1 , este resultado se contradice con los autores $5,8,10$ los cuales encontraron que el promedio de consumo en un día típico es alto (11-20 cigarrillos respectivamente), lo que puede deberse a que este estudio se realizó en personas pertenecientes al área de la salud y por lo tanto puede ser que tenga un compromiso social ante la problemática de las adicciones y esto los lleva a consumir menos cigarrillos.

La edad de inicio de consumo de tabaco del personal de salud es de 13 años lo cual se contrapone por lo obtenido en investigaciones ${ }^{5,10}$ donde el inicio en estos estudios fue entre 18 y 20 años, esto podría ser que para los participantes del estudio se dio en la etapa de la adolescencia en la que existe mayor vulnerabilidad para experimentar el consumo de sustancias ilícitas.

En lo que corresponde al índice de valores instrumentales reportaron media más alta $(\bar{X}=72.99, D E=13.43)$ en comparación del índice de los valores terminales $(\bar{X}=66.87$, $D E=11.82)$, estos son iguales a los estudios ${ }^{15-17}$ donde los más altos son los instrumentales, una explicación posible sería que los comportamientos que las personas realizan van dirigidos alcanzar metas en el transcurso de la vida.

En lo que concierne a la prevalencia del consumo de tabaco en el personal de salud de primer nivel de atención, la prevalencia global es de $73.3 \%$, lápsica 3\%, actual 4.7\%, instantánea $2.4 \%$, este reporte es menor a la de la población general de acuerdo a los resultados obtenidos en la Encuesta Nacional de Adicciones $2011^{5}$.

En relación con los valores instrumentales más frecuentes en el personal de salud que consume, es el valor de ser capaz (efectivo y eficiente), este resultado es congruente con algunos autores ${ }^{15-18}$, situación que podría ser a que no importa la edad que la persona tenga sino el hecho de experimentar nuevas vivencias que generan una satisfacción en distintos momentos de la vida.

De acuerdo con los valores terminales de los participantes de esta muestra, el que más sobresalió fue el valor de una vida excitante el resultado difiere con otras investigaciones ${ }^{15-19}$ donde el valor atrevido (en busca de aventuras y riesgos) es el más importante. Una explicación podría ser referente a la edad que las poblaciones son distintas y el adolescente quiere emociones, mientras que el personal de salud tiene a su cargo responsabilidades que lo compromete a realizar su trabajo de forma ordenada. 
En lo que corresponde a la relación de los valores con el consumo de tabaco en el personal de salud en esta muestra no se encontró relación, esto es similar con los estudios realizados ${ }^{15-19}$. Una explicación sería que el hecho de consumir alguna sustancia no genera comportamientos de lo que está bien o mal, ya que el consumo les permite sentirse bien consigo mismo y relajados de las exigencias generales.

\section{CONCLUSIÓN}

En esta muestra el 22\% consume tabaco, la edad de inicio es a los 13 años consumiendo 1 cigarrillo en un día típico, el género que presenta mayor frecuencia de consumo es el masculino y personal médico. La prevalencia en relación al consumo de tabaco en el personal de salud en toda su vida fue del $73.3 \%$, en el último año lápsica $3 \%$, actual $4.7 \%$ e instantánea $2.4 \%$.

Los valores instrumentales de mayor importancia del personal de salud que consumen tabaco son ser capaz, el de menor importancia tener influencia en las personas. Con respecto a los valores terminales fue experiencias estimulantes. De acuerdo con la relación de los valores terminales e instrumentales no se encontró relación, los valores no influyen en el consumo de tabaco del personal de salud. Hay limitaciones, ya que existe poca evidencia respecto a los valores en el personal de salud donde aborde dicha temática.

\section{REFERENCIAS}

1. Organización Mundial de la Salud. Tabaco. Datos y cifras. Ginebra: OMS; 2018 [acceso en 2018 jun 10]. Disponible en: http://www.who.int/es/news-room/factsheets/detail/tobacco

2. Organización Panamericana de la Salud. Informe sobre el control del tabaco en la Región de las Américas, 2018. Washington: OPAS; 2018 [acceso en 2018 jun 10]. Disponible en: http://iris.paho.org/xmlui/handle/123456789/49237

3. Organización Panamericana de la Salud. Informe sobre el Control del Tabaco en la Región de las Américas. A 10 años del Convenio Marco de la Organización Mundial de la Salud para el Control del Tabaco. Washington: OPAS; 2016 [acceso en 2018 jun 10]. Disponible en: 
http: / /iris.paho.org/xmlui/bitstream/handle/123456789/28380/9789275318867_spa. pdf

4.. Instituto Nacional de Salud Pública. Encuesta Nacional de Adicciones 2011: tabaco. Cuernavaca, México., 2013 [acceso en 2018 jun 10] Disponible en: https://www.insp.mx/images/stories/Produccion/pdf/130911_ENAtabaco.pdf

5. Instituto Nacional de Salud Pública. Encuesta Nacional de Salud y Nutrición. Resultados por entidad federativa, Puebla 2012. Cuernavaca, México; 2013 [acceso en 2018 jun 20] Disponible en: http://studylib.es/doc/5612279/puebla---encuestanacional-de-salud-y-nutrici\%C3\%B3n-2012

6. Pérez SV, Ferreira PS, Pillon SC. Tabaquismo en las enfermeras de un hospital nacional de Lima, Perú. Rev latinoam enferm. 2010; 13(esp):550-6.

7. Reyes UJ, Burón PA, Sala SM, Serra PC, Diaconu D, Macia GF. Evolución del consumo de tabaco de trabajadores de un hospital de Cataluña. Rev Esp Salud Pública. 2013; 87(4):407-17.

8. Tamayo CC, Alejo PJ, Chambi HI. Relación entre estrés y hábitos de fumar en personal de salud, ciudad de la Paz Bolivia, 2014. Cuad Hosp Clín. 2015; 56(1):27-35.

9. Hernández PJ, Jadraque JP, Sánchez CA, Gómez AF. Prevalencia de consumo de tabaco entre los trabajadores del área de la salud de Isla de la Palma. Med Gen y fam. 2015; 4(3):63-7.

10. Maya MJ. Tabaquismo en trabajadores de la salud. Medigraphic. 2007; 75(4):2 pantalla.

11. Alonso MC, López K, Facundo AG. Factores de Riesgo y Consumo de Drogas en adolescentes de secundaria un estudio multiregional: El caso México. Monterrey Nuevo León, 2012. p 77-85.

12. Santana FK, Rey FY. Necesidad de la formación de los valores en los profesionales de la salud. Medisan. 2015; 19(3): 297-99.

13. Cayon A, Pérez E. Estructura de Valores de Schwartz en el personal directivo universitario privado. Talos. 2008; 10(3): 403-17.

14. Puente LAR, Alonso-Castillo BA, Alonso-Castillo MM, Alonso-Castillo MTJ, OlivaRodrígues NN, Armendáriz-García NA. Valores terminales, valores instrumentales y consumo de alcohol y tabaco es estudiantes de preparatoria. Enferm Comun. 2015; $11(1)$. 
15. Ocampos LF. Valores Terminales e Instrumentales y Consumo y no Consumo de Alcohol y Tabaco en Estudiantes de Enfermería [tesis]. Monterrey: Universidad Autónoma de Nuevo León; 2014.

16. Martínez MR. Valores y su relación con el consumo de alcohol y tabaco en adolescentes de preparatoria [tesis]. Monterrey: Universidad Autónoma de Nuevo León; 2014. 107 p.

17. Avendaño MGR. Valores, Consumo de alcohol y tabaco en estudiantes de bachillerato. [tesis]. Monterrey: Universidad Autónoma de Nuevo León; 2014. 106 p.

18. Burns N, Grove SK. Investigación en enfermería. Barcelona: Manual Moderno; 2012.

19. Secretaría de Salud (MX). Reglamento de la Ley General de Salud en Materia de Investigación para la Salud. México: Secretaría de Salud; 2014.

Conflito de interesses: Os autores declaram não haver conflito de interesses.

\section{Participação dos autores:}

- Concepção: Tenahua-Quitl I, Morales-Castillo FA, Torres-Reyes A.

- Desenvolvimento do estudo: Sánchez-Serrano CS, Tenahua-Quitl I.

- Redação e revisão: De Avila-Arroyo ML, Xicali-Morales N.

Como citar este artigo: Sánches-Serrano C, Tenahua-Quitl I, XicaliMorales N, Morales-Castillo FA, De Avila-Arroyo ML, Torres-Reyes A. Valores terminales, instrumentales y consumo de tabaco en el personal de salud. Journal Health NPEPS. 2018 jul-dez; 3(2):402-412. 